\title{
A Novel Device for Safe Trocar Insertion in Laparoscopic Surgery Based on the Insertion Force Characteristics
}

\author{
Junpeng Sun*, Kotaro Tadano \\ Department of Engineering, Tokyo Institute of Technology, 226-8503, Japan. \\ * Corresponding author. Tel.: +81 045-924-5032; email: junpengsun@hotmail.com \\ Manuscript submitted July 10, 2018; accepted December 15, 2018. \\ doi: 10.17706/ijbbb.2019.9.1.1-8
}

\begin{abstract}
Overshooting of trocar insertion in laparoscopic surgery has been extensively cited as a major cause of surgical injuries. Besides, the complexity and large insertion force of the current handheld insertion method also increase the risk of overshooting and difficulty for novices or female operators. Hence, we developed this trocar insertion device to make trocar insertion easier and safer. The device is designed easy to handle and trocar-assembling. And we used negative pressure and pneumatic cylinders to lift the abdominal wall and two motors to drive the trocar insertion. In addition, we interpreted the characteristics of insertion force and proposed an algorithm to detect whether it has penetrated out. With this algorithm the device can stop inserting automatically and immediately. The development of this device realized the automation of trocar insertion. It reduced the demand for operators and improved the safety of this procedure.
\end{abstract}

Key words: Trocar insertion device, automatic stop, overshooting, force characteristics, laparoscopic surgery.

\section{Introduction}

Trocar insertion is the first step in the majority of minimally invasive surgical procedures. In this procedure, a trocar, which is a long sharp tool, is penetrated through the abdominal wall of patients to create necessary ports. Then, operators can insert other medical tools such as a laparoscope, forceps through the trocar to take the operation [1]. Usually, 3-5 ports are required in a laparoscopic surgery. Currently, majority of trocar insertion have to be done by two operators at the same time. One person lifts the abdominal wall and the other operator inserts the trocar. According to previous researches [2], inappropriate lifting of the abdominal wall would produce local pressure to cause subcutaneous bleeding, which influenced postoperative beauty. What the worst is that overshooting of the trocar may injury organs or arteries under the abdominal wall. It leads to complications such as hemodynamic shock, respiratory arrest and even death [2-4]. Approximately 75,000 inadvertent trocar insertion occur annually in the U.S. alone [2]-[4]. Currently techniques for accessing specific regions of the body with trocars, needles and catheters typically involve blind guidance where the physician or nurse relies entirely on tactile feedback and experience. The operator must sense when the trocar (or needle) enters the target space and discontinue advancement. Although some manufacturers improved the design of the trocar, which allows placing the laparoscope into the trocar to help operators get visual feedback [5], [6], recognition of anatomical landmarks and skilled operation are still necessary for operators. In addition, with the growing problem of obesity, the skin-to-target distance is increasingly variable, and inserting the trocar safely 
becomes more difficult for operators, especially for the novices [7].

Related works about improvement of trocar insertion mainly can be divided to two branches as follows:

One branch is to improve the design of the trocar or attach other micro devices or sensors to the trocar. Different degrees of improvement and related researches were conducted. In addition to the spring-loaded trocar and the bladeless trocar which allows placing the laparoscopy inside mentioned above, ultrasound and active sensing systems on the tips of the trocar (or needles) were developed. The other branch is to develop trocar-insertion training system for operators. Many researchers focus on providing a training system or platform for operators to help them exercise. For example, a VR (virtual reality) training system [8], [9] was developed by Sakaguchi's group. Operators can do insertion simulation on the platform. And a master-slave assisted robot [10], [11] have been developed by Ashiwad Chowriappa.

In this paper, we developed an automatic trocar insertion device, which can insert usual trocar products in the market. What's more interesting is that negative pressure and pneumatic cylinders are used to lift the abdominal wall. Because of that, this device allows one operator finish the insertion work by himself or herself. Besides, based on the interpretation for insertion force characteristics, we proposed an algorithm for automatic stop. Upon the trocar has penetrated out of the abdominal wall, this device can discontinue inserting timely to avoid overshooting. And finally, we verified its practicality by an exvivo porcine experiment. We evaluated the insertion effect with this device by recording exposure length of trocar tips when it stopped.

This paper is arranged as follows, prototype of the trocar insertion device is described in section 2. It includes the description of device design in section 2.1 and the control methods for lifting the abdominal wall and inserting trocars in section 2.2. In section 3, based on related researches about mechanics of dynamic needle insertion [12], [13] and the multilayer structure of the abdominal wall, we interpreted the insertion force characteristics. And combined with the design of the device, we proposed an algorithm for automatic stopping. In section 4 , we did exvivo porcine experiments to verify the usability of this device. In experiments, we confirmed the automatic stop algorithm we proposed by observing the driving force of cylinders. In addition, we evaluated the trocar insertion effect using this device by recording exposure length of the trocar when it stopped. Finally conclusions appear in section 5. This device allows operators insert the trocar more safely, faster and more conveniently.

\section{Overview of Trocar Insertion Device and the Controller}

\subsection{Trocar Insertion Device}

Prototype model of this device is shown in Fig. 1. It has a pistol shape and appropriate size $(282 \mathrm{~mm}$ * $122 \mathrm{~mm} * 237 \mathrm{~mm}$ ), which allows operators handing easily. The trocar is fixed along the central axis. This device consists of two motor combinations, the first one is assembled inside the handle, and this motor drives the trocar inserting forward through the ball screw, belt and belt wheels. The other motor is assembled behind the trocar, which drives the trocar rotating along the central axis (red arrows in Fig. 1).

In order to lift and hold the abdominal wall during insertion, we draw on the method of cupping, which is proved that there is no harm to the human body in medicine. To make the lifting relatively uniform and reliably, six pneumatic cylinders are distributed around the trocar. The tips of cylinder rods are connected to suckers. And suckers connect to a vacuum generator. During insertion, suckers can be fixed to the surface of the abdominal wall by negative pressure produced by the vacuum generator (yellow arrows in Fig. 1), and the air cylinders lift the abdominal wall to restrain the deformation caused by insertion (blue arrows in Fig. 1). There are four wire-encoders assembled near the air cylinders. They are used to measure the displacement of the cylinder rods for the cylinder control, we will describe them in detail in the sections that follow. 
In detail of this device, it adopts a combination design of trocar fixed and main body shown in Figure above. It not only allows this device usable for various trocar products in the market by changing trocar fixed, but also makes it convenient when loading the trocar before insertion and unloading the trocar after finished. In addition, as the abdominal wall is uneven, we added ball joints between suckers and air cylinder rods to ensure suckers can be fixed to the abdominal wall more fitly.

\subsection{Controllers}

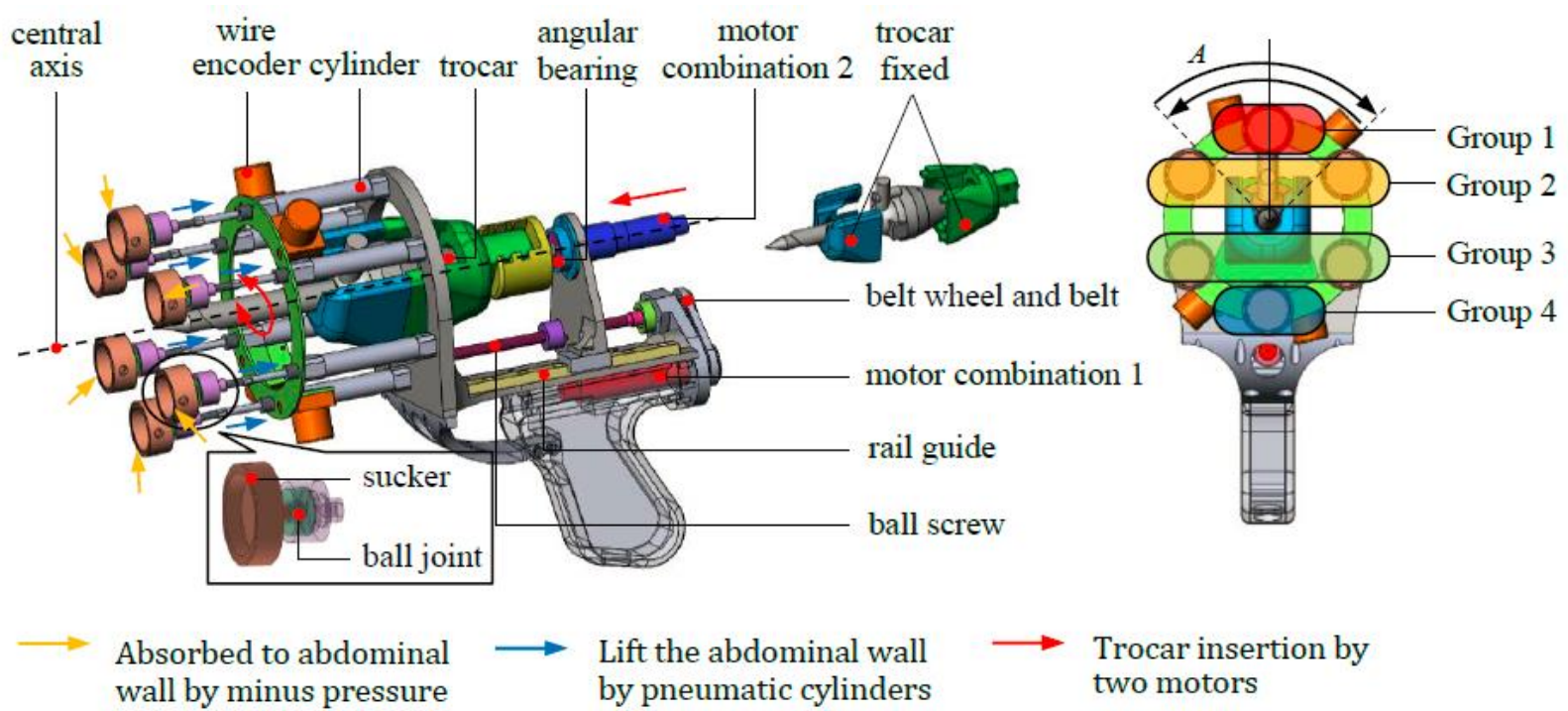

Fig. 1. Trocar insertion device.

Suckers are connected to a vacuum generator, which produced $-80 \mathrm{kPa}$ constantly. According to an easy calculation, the adhesive force of every sucker is about $62.17 \mathrm{~N}$.

Motor combination 1 in Fig. 1 drives the trocar inserting forwards. Under a positional PID controller, the trocar can inserts forwards at a constant speed of $0-12 \mathrm{~mm} / \mathrm{s}$.

Motor combination 2 in Fig. 1 drives the trocar rotation during it forwards. Under a positional PID controller, it rotates, whose amplitude $A$ is $\pi / 4$, period $T$ is $1 \mathrm{~Hz}$, shown in Fig. 1 right.

We separated the air cylinders to four groups from up to down as is shown in Fig. 1 right. In every group, one servo valve drove one or two air cylinders, two pressure sensors measured the air pressure in two chambers of cylinders, and a wire encoder measured the displacement the rod moved. Every group is under impedance control independently. The block diagram of the air cylinder control is shown in Fig. 2. There is a force feedback as an inner loop. $F_{d r_{-} \text {ref }}$ represents the reference driving force of the air cylinder, which can be expressed as follow,

$$
F_{d r_{-} r e f}=K_{p p}\left(q_{r e f}-q\right)+K_{p d} \frac{d\left(q_{r e f}-q\right)}{d t}
$$

where, $q_{\text {ref }}$ represents the reference value of the displacement of the cylinder rod. In the later experiments, we set up $q_{r e f}$ to a constant value so that the cylinders can lift the abdominal wall during insertion. $q$ represents the actual displacement of the cylinder rod, which is measured by wire encoders.

$F_{d r}$ represents the actual driving force of the air cylinder, which is calculated by the air pressure of the two chambers of the air cylinder. The air pressures are sensed by pressure sensors. And $K_{p p}, K_{p d}, K_{a p}$, $K_{a i}$ are coefficients of the controller. In Fig. 2, the insertion force $F_{\text {insert }}$ is treated as a disturbance. Thus, 
$F_{d r}$ reflects the insertion force.

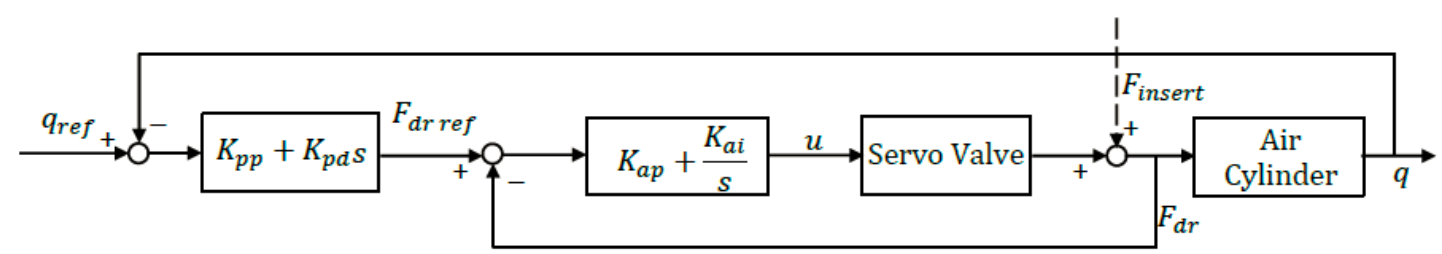

Fig. 2. Block diagram of pneumatic cylinders impedance control.

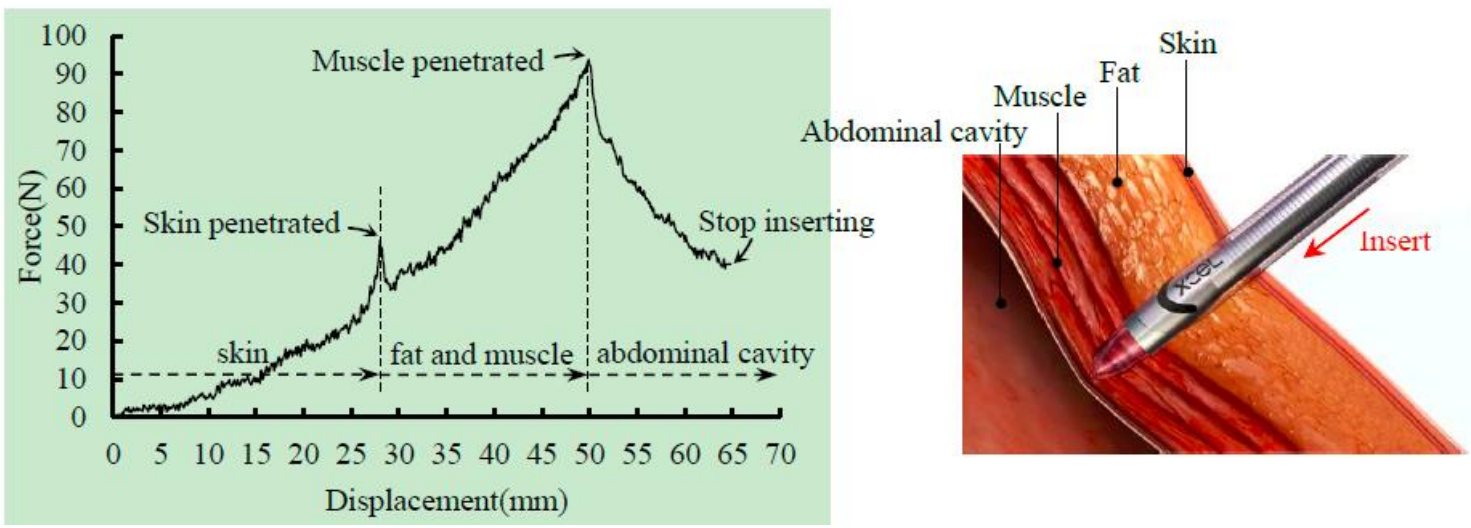

a. Insertion force vs. absolute trocar displacement since contact. b. Multi-layers of the abdominal wall

Fig. 3. Characteristics of trocar.

\section{Force Characteristics during Insertion and Algorithm of Automatic Stop}

In order to achieve the automatic stopping upon penetrated out, we need to find out when the trocar penetrated out of the abdominal wall.

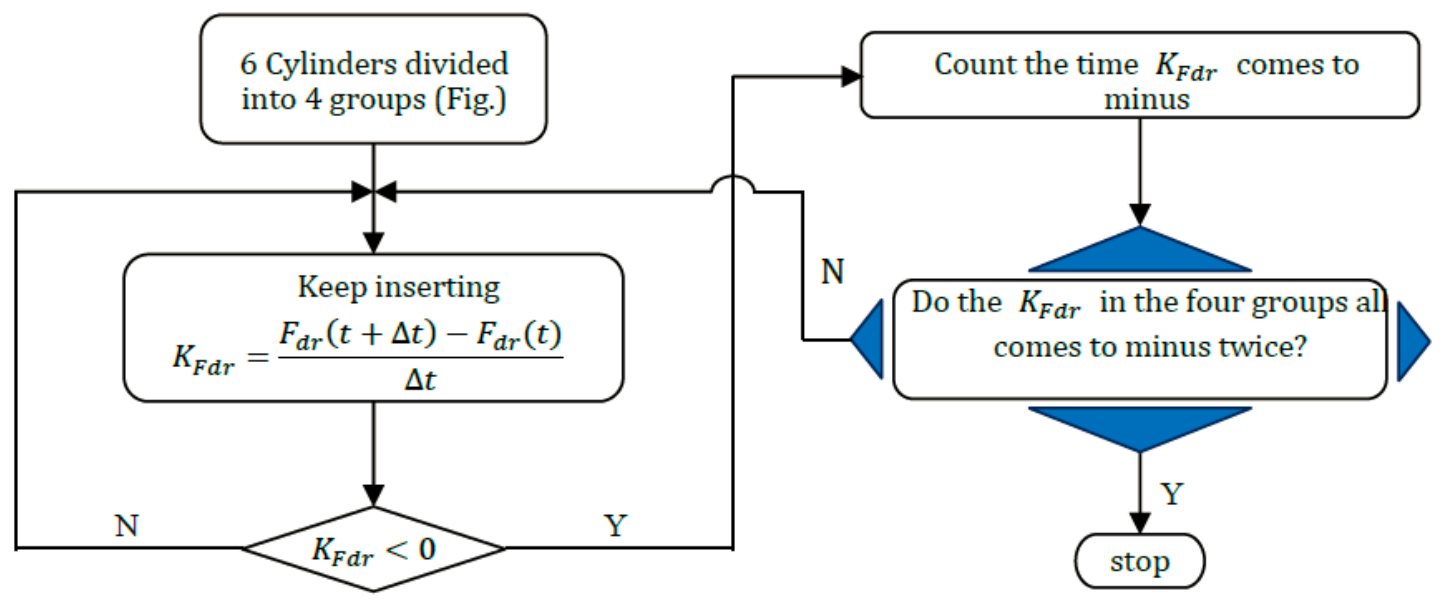

Fig. 4. Flowchart of the automatic stopping algorithm.

Fig. 3 a shows a typical force-displacement responses measured by a force-measuring platform we developed, during the insertion of a rigid trocar through a porcine abdominal wall. Because the abdominal wall has a multi-layers structure (Fig. 3. b) and the skin is harder than fat, a force rupture appears when the skin is penetrated out. Then, because fat is softer than muscle, there is no force rupture can be observed when fat is penetrated out. The muscle is the bottom layer and the hardest, when it is penetrated out, the 
second rupture appears. Need to emphasize that the size of ruptures may change with many factors, such as type of the trocar, insertion speed, patients' individual difference, etc. but it does not change that the first rupture responses to the skin is penetrated out, the second rupture responses to the whole abdominal wall is penetrated out. It is determined by the multi-layer structure and the hardness of each layer.

Based on this force characteristics, we proposed the algorithm that the trocar stops inserting when it detects the second rupture of the insertion force. During insertion, we calculate the average slope of the insertion force in a certain sampling period of 100 samples. When $K_{F d r}$ (expressed in Fig. 4) comes to negative at the second time, it is identified as the point where the trocar penetrated out of the abdominal wall. In actual sampling and calculation, there are noises to lead to misjudgment. Thus, we design it that only if the $K_{F d r}$ of the four groups all comes to negative, it will be counted once. The synchronously judgment of the four groups can effectively filter force fluctuation or noises. The algorithm is shown in Fig. 4.

\section{Exvivo Insertion Experiment}

In order to verify the usability of this device, we had an exvivo porcine experiment as is shown in Fig. 5. The constant insertion speed is set up to $10 \mathrm{~mm} / \mathrm{s}$, and the amplitude and the period of the trocar rotation, $A$ and $T$ is set up to $\pi / 4$ and $1 \mathrm{sec}$. In experiment, the suckers can be fixed onto the surface of the abdominal wall by negative pressure successfully. Driven by the two motors, the trocar can penetrate out of the abdomen and the pneumatic cylinders can lift the abdomen to restrain the deformation successfully. Measurements in every group during experiments are listed in Fig. 6. It consists of air driving forces of the cylinders, the $K_{F d r}$ calculated in every group and the displacement of the cylinder rods. In order to make it easy to see, we added the absolute displacement the trocar inserted since it contacted to the abdominal wall by red lines in the air driving force figures. It could be observed that the two ruptures of the insertion force were reflected by the air driving forces. And when the trocar penetrated out of the abdominal wall, the force passed the max peak and decreased. But because the device stopped inserting immediately, the driving force kept in a stable value instead of going on decreasing like the force in Fig. 3. A.
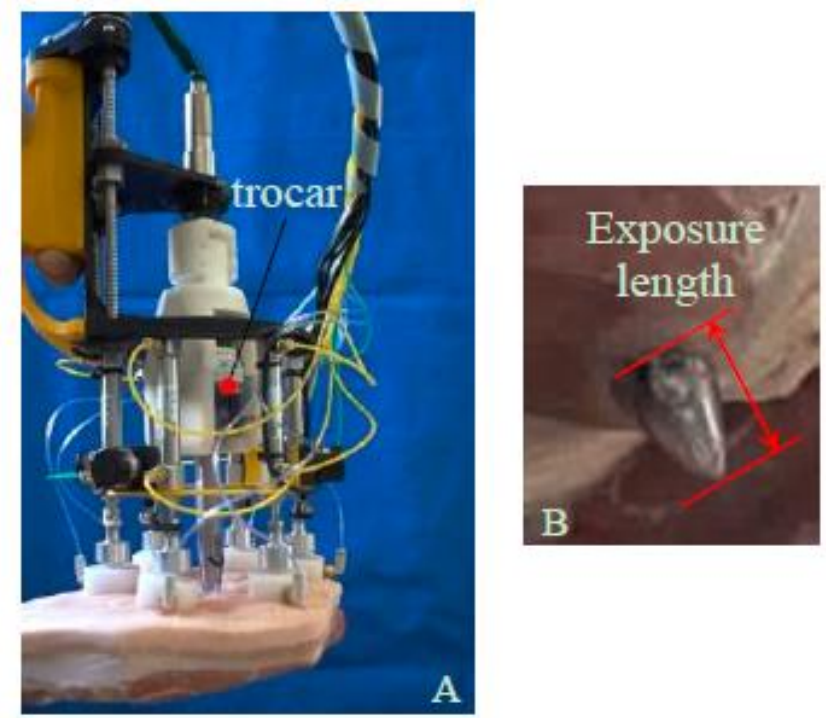

Fig. 5. Insert porcine abdominal wall by the device.

In addition, it can be observed in the graph of the $K_{F d r}$ in group 3 (2nd Row, 3rd Column in Fig. 6) that the slope has come to negative once before the force comes to the first rupture. It was caused be noises and 
fluctuation of force. According to the algorithm mentioned above, this noise was filtered successfully to avoid misjudgment. This is also a reason why we separated the six pneumatic cylinders to four groups as shown in Fig. 6.

Group 1

Group 2

Group 3

Group 4
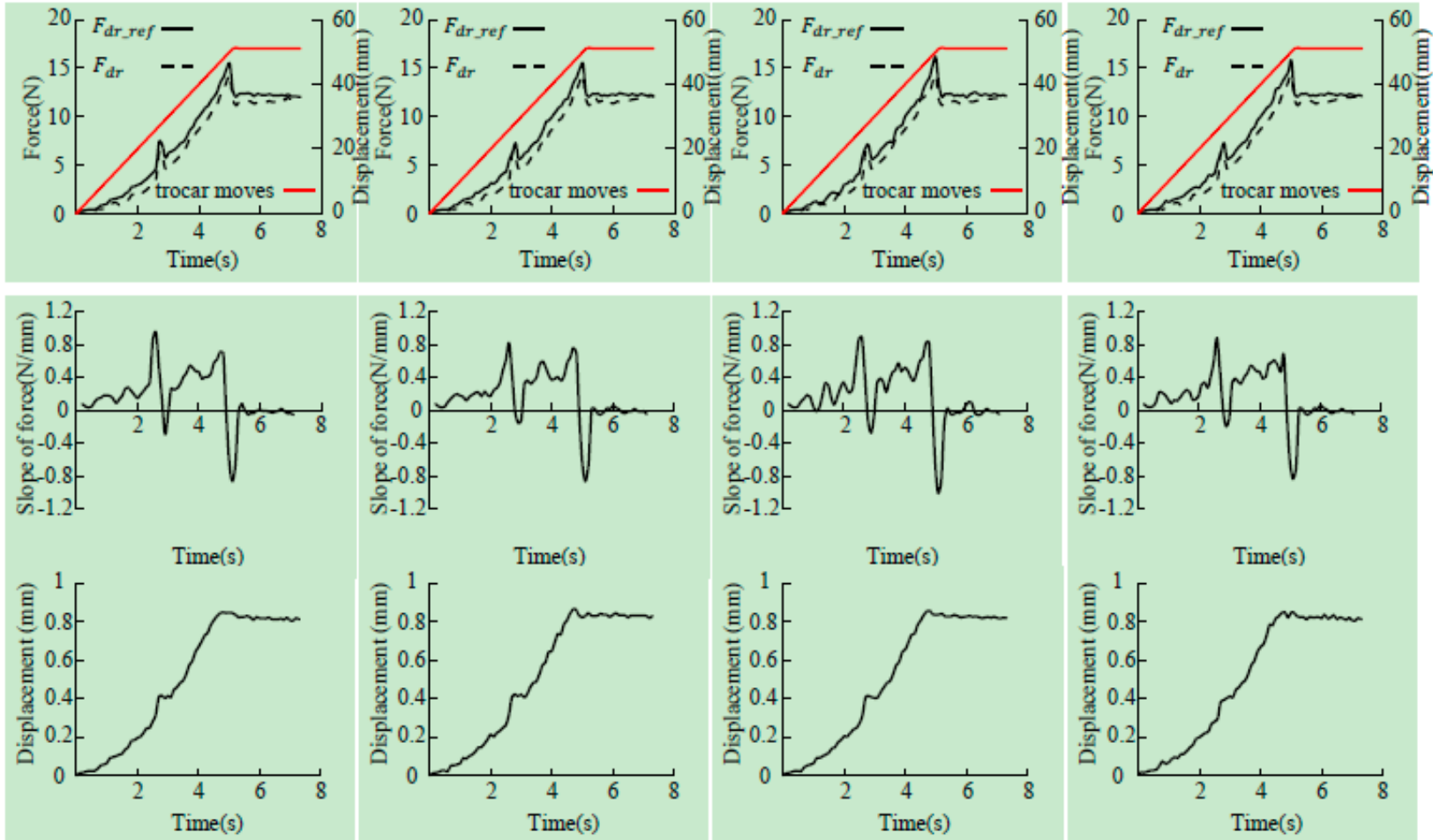

Fig. 6. Measurements in the four groups.

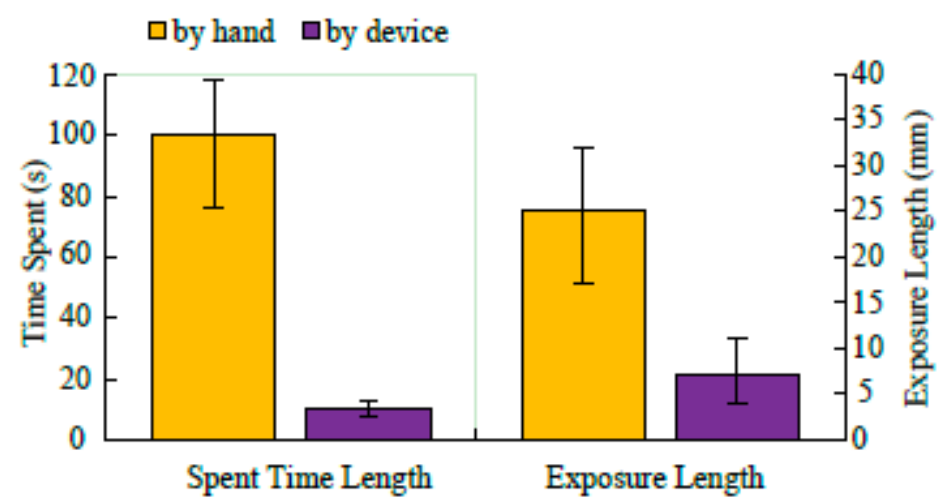

Fig. 7. Evaluation of insertion with and without this device.

We also evaluated the effect of this device. We repeated the insertion experiment with this device and recorded the time insertion spent and the exposure length of the penetrated trocar. And according to clinical statistics [3-8], we listed the comparison as is shown in Fig. 7. It can been observed that insertion with this device considerably reduced the operation time and it has shorter exposure length, which means safer, than insertion by hands. Not only that, this device eliminated difference of operation done by experienced operators and novices.

\section{Conclusion}


In this paper, we developed an automatic trocar insertion device in response to the overshoot of insertion in laparoscopic surgery. The insertion device is easy to handle and it realizes the combination of lifting the abdominal wall and inserting the trocar. That makes it possible that one surgeon finished the insertion procedure by only himself or herself. Based on the insertion force characteristics, we proposed an algorithm for automatic stop upon penetrated out. In exvivo porcine experiments, it proves that this device can allows operators to insert the trocar faster and safer. It effectively improved the convenience and safety of the trocar insertion procedure.

In order to achieve clinical application, there are still some questions to solve. For example, sterilization, miniaturization and applicability to special clinical cases such as peritoneal adhesions. Those are also the points we will focus on in our future research.

\section{References}

[1] Kucklick, T. (2006). The Medical Device R\&D Handbook. Boca Raton: Taylor \& Francis.

[2] Sharp, H. T., et al. (2002). Complications associated with optical-access laparoscopic trocar. Obstet. Gynecol., 99(4), 553-555.

[3] Krishnakumar, S., \& Tambe, P. (2009). Entry complications in laparoscopic surgery. J. Gynec. Endosc. Surg., 1(1), 4-11.

[4] Rocco, O., Pietro, P., \& Flavio, L. (2003). Needle and trocar injuries in diagnostic laparoscopic under local anesthesia: what is the true incidence of these complications? J. Laparoendosc. Adv. Surg. Tech. A, 13(3), 181-184.

[5] Brown, J. A., et al. (2005). Optical-access visual obturator trocar entry into desufflated abdomen during laparoscopic; assessment after 96 cases. J. Endourol., 19(7), 853-855.

[6] Vygotsky, L. S. (1991). Genesis of the higher mental functions. Learning to Think, 32-41. London: Routledge.

[7] Semm, K., \& Semm, I. (1999). Safe insertion of trocars and the Veress needle using standard equipment and the 11 security steps. Gynaecol. Endosc., 8(6), 339-347.

[8] Sakaguchi, M., et al. (2006). Development of virtual trocar insertion training system for endoscopic surgery. Int. J. Comput. Assist. Radiol. Surg., 1(1), 159-161.

[9] Sakaguchi, M., et al. (2008). Measurement of trocar inserting motion for endoscopic surgery and development of force feedback device for training. Proceedings of the 5th Int. Conf. Ubiquitous Robots and Ambient Intelligence (pp. 643-646).

[10] Chowriappa, A., et al. A predictive model for haptic assistance in robot assisted trocar insertion. Proceedings of IEEE World Haptics Conference 2013.

[11] Yen, P., et al. (1996). A telemanipulator system as an assistant and training tool for penetrating soft tissue. Mechatronics, 6(4), 423-436.

[12] Okamura, A. M., et al. (2004). Force modeling for needle insertion into soft tissue. IEEE Trans. Biomed. Eng., 51(10), 1707-1716.

[13] Mahvash, M., \& Dupont, P. E. (2010). Mechanics of dynamic needle insertion into a biological material. IEEE Trans. Biomed. Eng., 57(4), 934-943.

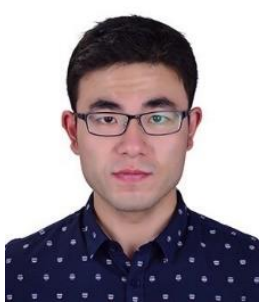

Junpeng Sun was born in China on November 20, 1989. He got the bachelor of mechanical engineering from China Agriculture University in 2012; the master degree in ME from Beihang University, China 2015. He is studying forwards a doctoral degree in Tokyo Institute of Technology, Japan now. His research focuses on the development of biomedical device. 


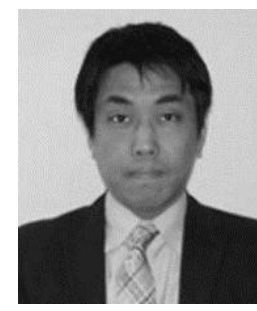

Kotaro Tadano received the B.S. degree in physics and the M.S. and Dr. Eng. Dgrees in mechanical engineering from Tokyo Institute of Technology, Yokohama Japan in 2003, 2005 and 2007 respectively. He is currently working as an associate professor in Tokyo Institute of Technology. His research focuses on surgical assistant robot, haptic, pneumatic system and teleoperation. He received an IEEE Robotics and Automation Society Japan Chapter Young Award and is a member of the IEEE Robotics and Automation Society. 